\title{
MODELLING OF SUBMERGED OPTICAL REMOTE CORRESPONDENCES WITH LOW LOSSES
}

\author{
BALAJI K ${ }^{1}$, R. NITHYA ${ }^{2}$, Dr. S. GANESAN ${ }^{3}$, N. NISHAVITHRI ${ }^{4}$ \& Dr. S. SAKTHIVEL MURUGAN ${ }^{5}$ \\ ${ }^{1,2,4}$ Assistant Professor, Department of Electronics and Communication Engineering, \\ Mailam Engineering College, Mailam, India
}

${ }^{3}$ Professor \& Head, Department of Electronics and Communication Engineering, Mailam Engineering College, Mailam, India

${ }^{5}$ Associate Professor, Department of Electronics and Communication Engineering, SSN College of Engineering, India

ABSTRACT
Submerged optical remote correspondences (SORC) have increased a significant enthusiasm during the most recent ages
as elective methods for high speed internet economical undersea interchanges. SORC present various likenesses
contrasted with free space optical (FSO) correspondences or laser outpost connections for the most part because of the
way Use optical frequencies to move secure data between point-to - point connections committed. High information rates
can be achieved through the utilization of appropriate frequencies. Some ongoing work has shown that outpost
connections can be made in moderate quantities. In research facilities study, transmissions of some Mbps were recognized
through use of a recreated medium with attributes such as maritime waters.
$\quad$ SORC systems were also shown to operate at high information levels for medium distances of up to 100 meters.
Nonetheless, it is actually not accessible as a modern item and test bed figures have mostly been taken into account until
now in water test tanks. Therefore, large work is expected sooner rather than later, which is necessary to further expose
covered-up' optical capacity to move outpost signs at greater distances. The present work summarizes continuing
progress in demonstrative channels and the research and structure framework in the SORC area.
KEYWORDS: Submerged Optical Remote Correspondences (SORC), Channel Demonstrating, Framework Investigation,
Test-Bed Estimations

Received: Jun 01, 2020; Accepted: Jun 19, 2020; Published: Jul 18, 2020; Paper Id.: IJMPERDJUN2020378

\section{INTRODUCTION}

The current work sums up the ongoing advances in channel displaying and framework investigation and plan in the zone of submerged optical remote correspondences (SORC). SORC have increased a significant enthusiasm during the most recent years as an elective methods for outpost modest submarine correspondences.

The innovation that is generally utilized these days among jumpers, ships, and so on is basically founded on acoustic wave transmission. In any case, it is confined to a few several kbps at scopes of a couple of kilometers, underpins moderate information rates, and bothers marine warm blooded creatures, for example, dolphins and whales. Then again, customary electromagnetic (EM) waves are profoundly lessened in water because of both retention and dispersing. Along these lines, they can be utilized distinctly for generally short-range real-time applications in the request for a couple of centimeters to a couple of meters. Fig.1 describes the importance of underwater and ways of communication in underwater. 


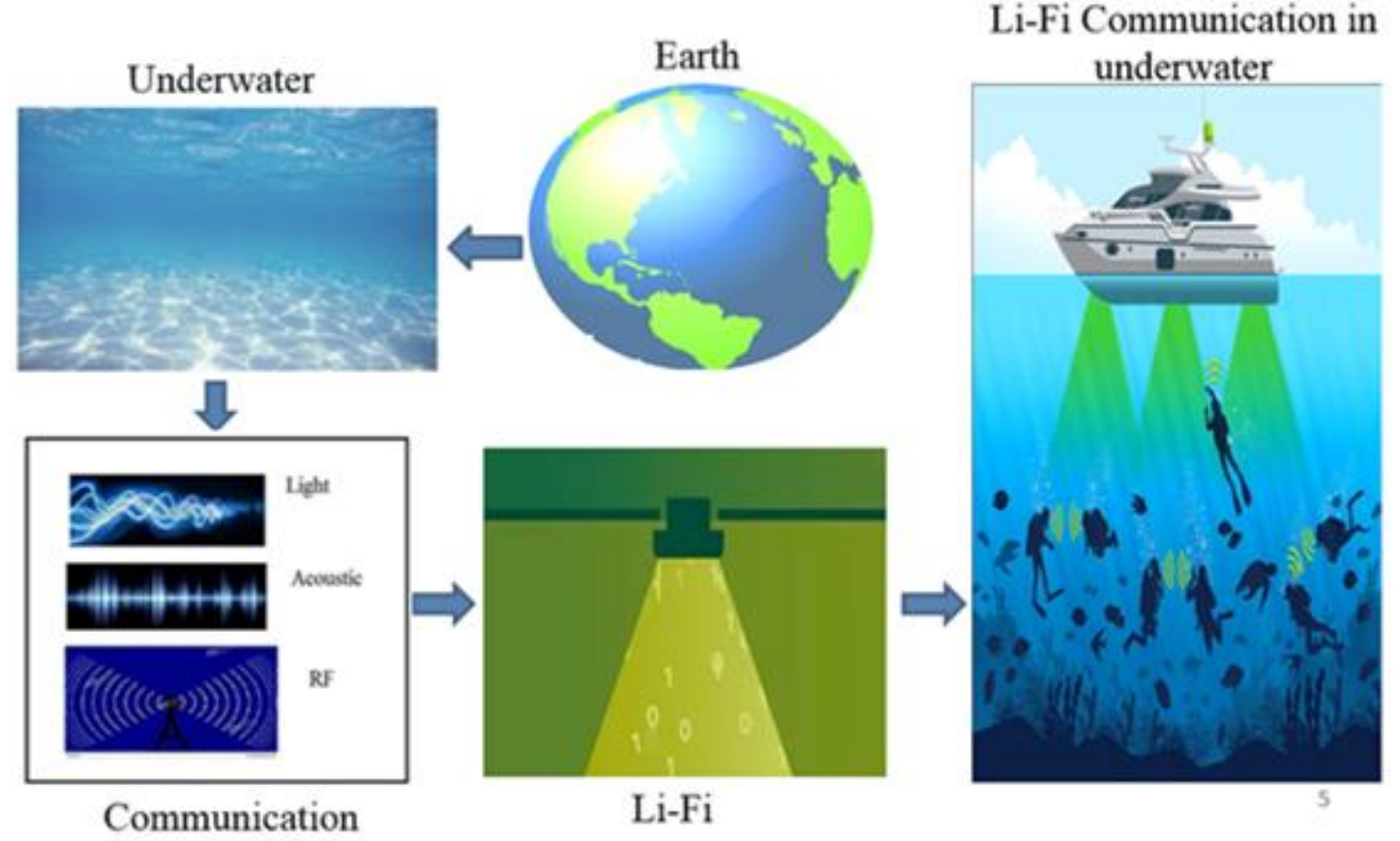

Figure 1: Li-Fi Communication in Underwater.

EM waves in the noticeable range, for instance, optical signals somewhere in the range of 400 and $700 \mathrm{~nm}$, proliferate quicker in water than acoustic ones. In any case, the visual transmitters and recipients must be situated at a short separation, since choppiness and multiscattering impacts essentially fall apart the presentation. Chromatic scattering additionally causes fleeting expanding of the optical heartbeats. Rather than the above debilitations, seawater shows a diminished ingestion in the blue/green locale of the noticeable range. Consequently, utilizing appropriate frequencies, high information rates can be accomplished. In addition, some ongoing works demonstrated that outpost connections can be accomplished over moderate extents. Transmissions of a few Mbps have been acknowledged in lab tries by utilizing a mimicked sea-going medium with dissipating attributes like maritime waters. It was additionally shown that SORC systems are achievable to work at great information rates for medium separations up to a hundred meters.

SORC present various likenesses contrasted with free space optical (FSO) interchanges or laser satellite connections predominantly because of the way that they utilize optical frequencies to move secure data between committed point-to-point joins. It comprises a substitute and powerful transmission strategy for submerged correspondences, rather than the customary acoustic one. Be that as it may, it isn't as of now accessible as a mechanical item and for the most part test-bed estimations in water test tanks have been accounted for up until now. Thusly, broad research is normal sooner rather than later which is fundamental so as to additionally uncover the "covered up" capacities of optical range to move outpost signs at higher separations.

In the flow section, we plot the fundamental qualities of SORC channel models dependent on hypothetical and trial results, the marvels constraining their exhibition, and the key research preliminaries revealed so far for this novel part of optical remote frameworks. A short survey of acoustic and EM correspondence procedures is likewise introduced, which enables the perusers to more readily comprehend the various ways SORC works. 


\section{TRANSMISSION OF WAVES IN UNDERWATER MEDIUM}

\section{Acoustic Waves}

Until today, submerged transmission is accomplished by utilizing acoustic waves. This innovation bolsters, moderately, low information rates for medium separations and doesn't guarantee the connection security. Moreover, the data signal deferral is very expanded. It is a heritage innovation, and even it works at significant distances, it can just set up low speed transmissions. In this way, it is wrong for outpost transmission driven by the present and continuous correspondence requests.

Although it travels sound enough through the air, through water it goes much better. The sonic velocity in clear air is $340 \mathrm{~m} / \mathrm{s}$, which is almost many times smaller in water than the sound velocity of $1500 \mathrm{~m} / \mathrm{s}$. However, the speed of light and EM waves is much smaller.

At what speed sounds pass through the water, the temperature, weight and salinity of the water are profoundly dependent. As the acoustic waves travel through the water, the energy of the amphibian medium is absorbed and various structures such as heat can be converted[1]. The key impediments of underwater acoustic transmission are usually incorporated via spin-offs.

Way Loss: The lessening or way misfortune in a submerged channel is recurrence needy and communicated as [2]

$$
\mathrm{A}(\mathrm{l}, \mathrm{f})=\mathrm{l}^{\mathrm{k}} \alpha(\mathrm{f})^{1}
$$

The reason for the recurrence reliance is the vitality assimilation of the weight waves and the spreading misfortune that increments by the separation. The seawater ingestion coefficient is communicated by the entirety of substance unwinding procedures and assimilation from unadulterated water [3]

$$
\alpha=\frac{A_{1} P_{1} f_{1} f^{2}}{f_{1}^{2}+f^{2}}+\frac{A_{2} P_{2} f_{2} f^{2}}{f_{2}^{2}+f^{2}}+A_{a} P_{a} f^{2}
$$

Multipath: The spreading of the multipaths is a typical problem for submerged acoustic connections and leads to various sound signals reaching the collector. When everything is finished, reflected waves are taken from the transmitter from the base, from the surface, or from different items. Stable varieties of wave refraction with depth $[4,5]$ also occur. The receiver is also conscious of more than one heartbeat with an alternative stage of suitability and appearance [6]. The corresponding channel drive reaction is effectively articulated[7].

$$
C(\tau, t)=\sum_{p} A_{p}(t) \delta\left(\tau-\tau_{p}(t)\right)
$$

where $\mathrm{A}_{\mathrm{P}}(\mathrm{t})$ is the path amplitude and $\tau_{\mathrm{P}}(\mathrm{t})$ is the path delay, both time dependent

The main factors that affect many different paths are the transmission, depth, geometry, recurrence and sound speed profile. And even canals with a deference to the base of the sea, like the heading of the tide, have long multipaths, even though vertical canals extend shortly [8]. The acoustic sign can seriously decay due to the proliferation of multipaths because it causes a symbolic obstruction (ISI).

Commotion: Noise is made by machines which are being used or by transportation exercises as a rule in high rush hour gridlock zones. Another sort of clamor is encompassing commotion, which is generally identified with 
hydrodynamics brought about by the development of water starting with one spot then onto the next like flows or tides. Climate impacts on the ocean like breezes, downpours, storms, environmental choppiness, warm commotion, seismic, and organic marvels can likewise incite clamor $[2,8]$. The essential wellsprings of commotion in shallow waters appear to be made by ships and snapping shrimps [10].

Doppler Spread: Doppler recurrence spread is brought about by the relative movement between the transmitter and the recipient. ISI is likewise identified with Doppler spread generally in high information rate transmissions where adjoining images meddle at the collector. The Doppler impact can be assessed by the proportion $\mathrm{a}=\mathrm{Ur} / \mathrm{c}$ where $\mathrm{Ur}$ is the relative speed between the transmitter and the collector, and $\mathrm{c}$ is the sign engendering speed. The Doppler factor is about $\mathrm{a}=10^{-4}$ because of the generally low recurrence of the acoustic signals inside water $(1500 \mathrm{~m} / \mathrm{s})[2,8,11]$. Indeed, even at short ranges, the acoustic channel is restricted to low information rates under Mbps. For medium extents (1-10km), the information rate drops to roughly $10 \mathrm{kbps}$, and in the end at extremely long ranges $(>100 \mathrm{~km})$, the information rate is under $1 \mathrm{kbps}[9,12,13]$. Along these lines, the acoustic wave transmission can't fulfill the necessities of new requesting advancements due to the powerlessness to accomplish high information rate interchanges continuously activity.

\section{Electromagnetic Waves}

EM waves in the RF band can be utilized so as to accomplish quicker remote transmission. They are additionally unaffected by temperature and profundity. Lessening is the principle impact of water in all EM waves, because of both ingestion and dissipating. EM wave conduct in freshwater and seawater is very unique and that is because of the way that ocean water is a high-misfortune medium. Spread speed and the absorptive loss of EMwaves can be portrayed as elements of transporter recurrence $[14,15]$.

EM waves inside water are profoundly recurrence needy and corresponding to the square base of recurrence. This is the primary explanation behind utilizing low frequencies VLF and ELF. VLF band comprises of radio waves somewhere in the range of 3 and $30 \mathrm{kHz}$, can accomplish information paces of 300bit/s, and can infiltrate seawater at a profundity of $20 \mathrm{~m}$. Mythical person band $(3-300 \mathrm{~Hz})$ can enter seawater at profundities of several meters, something very valuable for correspondences between submarines.

To summarize, the primary impediments of EM waves are the huge recieving wire size required in freshwater and the high constriction for seawater [16]. Accessible business items for submerged in RF band can accomplish bit paces of 100bps for a scope of quite a few years of meters. 1-10Mbps inside $1 \mathrm{~m}$ territory has been accounted for, too [17].

\section{Optical Waves}

Optical remote correspondences are a moderately new innovation giving numerous genuine focal points, for example, the exceptionally high paces of information transmission, secure connections, little and lightsize of the handset segments, including openings, low establishment and operational expense, and no need of permitting charges and taxes, since the optical band is excluded from the broadcast communications guidelines [18]. Optical remote uses adjusted optical bars so as to build up short, medium or long correspondences. Shockingly, in light of the fact that the engendering medium is the free space, the exhibition and the unwavering quality of these frameworks rely essentially upon the climate conditions between the beneficiary and the transmitter $[19,20]$.

EM waves in the noticeable range (400-700nm) present an elective method to give outpost correspondences in the water. They spread quicker in water $(300,000,000 \mathrm{~m} / \mathrm{s})$ than the acoustic ones $(\sim 340 \mathrm{~m} / \mathrm{s}$ in air- $\sim 1500 \mathrm{~m} / \mathrm{s}$ in water), which 
is around multiple times quicker than sound goes through water. That is the fundamental motivation behind why they have increased an extensive enthusiasm during the most recent years to fill in as a outpost (10-100Mbps), safe (noninterceptable), and dependable supplement to inheritance acoustic submerged correspondences frameworks [6].

As a rule, optical signs are profoundly invested in water, and this is one of the principle hindrances; the other one is the optical dispersing by all the particles existing inside the ocean. Be that as it may, seawater shows a diminished assimilation in the blue/green area of the obvious range. In this way, utilizing reasonable frequencies, for example in the blue/green district, fast associations can be achieved by the sort of water (400-500nm for clear to 300-700nm for turbid water conditions). Least constriction is focused close to $0.460 \mu \mathrm{m}$ in clear waters and moves to higher qualities for grimy waters moving toward $0.540 \mu \mathrm{m}$ for waterfront waters $[21,22]$.

Generally, the force got $\mathrm{P}(\mathrm{z})$, given introductory force $\mathrm{P} 0$, proliferating through a mechanism of thickness $\mathrm{z}$ is assessed by the Beer's Law as follows

$\mathrm{P}(\mathrm{Z})=\mathrm{P} 0 \mathrm{e}-\mathrm{C}(\lambda) \mathrm{Z}$

$c(\lambda)$ is the attenuation coefficient expressing the total attenuation occurred by the propagation through the water.

As indicated by the Haltrin's model in Ref. [23], the all out lessening can be depicted as the total of assimilation and dissipating. For a totally retaining or totally dissipating medium, the complete lessening coefficient in Eq. (4) can be supplanted with the retention coefficient, that is, an, or dissipating coefficient, that is, b, separately. The item cz is likewise alluded as constriction length, and it contributes on the decrease of the got power by a factor of $\exp (-1)$, or $\sim 63 \%$ [24]. In view of the abovementioned, we have

$$
C(\lambda)=\alpha(\lambda)+\beta(\lambda)
$$

where $a(\lambda)$ is the absorption coefficient, $b(\lambda)$ is the scattering coefficient, and $\lambda$ is the wavelength.

Brew's Law gives a constrained relevance as it portrays just the lessening because of assimilation and single dissipating occasions. As a general rule, be that as it may, numerous instances of different dispersing may happen. Likewise it presumes that the source and collector are in accurate arrangement with one another, and it very well may be applied distinctly in Line-of-Sight (LOS) correspondence situations. Besides, Beer's Law overlooks transient scattering [25].

Progressively precise articulations need to consider the connection geometry. For example, accepting that the transmitter and recipient are situated in a LOS design, the got force can be assessed by [24]

$$
\mathrm{P}_{\mathrm{R}}(\mathrm{t}, \mathrm{r}, \mathrm{z})=\mathrm{P}_{\gamma}(\mathrm{t}) \mathrm{D}_{\gamma} \mathrm{L}_{\mathrm{w}}(\mathrm{t}, \mathrm{r}, \mathrm{z}) \mathrm{D}_{\mathrm{R}}
$$

Where $\mathrm{P}_{R}(t, r, z)$ is the gotten power subject to time $t$, sidelong relocation from the shaft hub r, and range $z, P_{T}(t)$ speaks to the transmitted force, $\mathrm{D}_{\mathrm{T}}$ is the gap and difference of the optical source, and $\mathrm{D}_{\mathrm{R}}$ is the photoreceiver gap and field of view. The channel misfortune term, $\mathrm{L}_{\mathrm{W}}(\mathrm{t}, \mathrm{r}, \mathrm{z})$, describes the spatial and worldly attributes of light proliferation in seawater. 


\section{SORC PROPAGATION PHENOMENA}

\section{Aquatic Medium Characteristics}

The oceanic medium contains right around 80 unique components, broke up or suspended in unadulterated water, with various focuses. Some of them are recorded beneath $[26,27]$

- Several salts have broken up, such as $\mathrm{NaCl}, \mathrm{MgCl} 2$ and so on, retaining explicit light at frequencies and prompting dispersion.

- Sand or metal oxides, for instance, that add intake and dissipation to both the detrital and mineral elements.

- Colored natural acids that hinder assimilation broke down, for example full and humic acids, primarily in the blue and bright frequencies.

- Bio-based issues such as pathogens, microscopic organisms, and natural scattering, particularly in the blue ghastly range.

- Chlorophyll-A phytoplankton, which firmly absorbs green light in the blue-red region.

- Since chlorophyll ingests the blue and red frequencies and the particles emphatically add to the dispersing coefficient, we can utilize its focus $\mathrm{C}$ (in $\mathrm{mg} / \mathrm{m} 3$ ) as the free parameter to figure the assimilation and dissipating coefficients $[23,28]$.

The specific sort of water assumes a huge job in the estimation of the measure of chlorophyll fixation and subsequently the measure of ingestion and dispersing for a particular geographic area. A characterization framework for the clearness of water types dependent on their ghastly optical weakening profundity $z_{k}=1 / k_{d}$ was proposed by Jerlov in 1968. This arrangement was made in the upper parts of the sea, and it depended on ghastly irradiance transmittance estimations [29]. The four significant water types that are normally alluded in the writing are the accompanying [30, 31]

- Pure profound sea waters cobalt blue where the retention is high and the dispersing coefficient is low.

- Clear ocean waters with higher dissipating because of many broke up particles.

- Near coasts sea waters with retention and dispersing because of planktonic issues, rubbish, and mineral segments.

- Harbor dim waters, which are very obliging for optical engendering due to broken down and in-suspension matters.

\section{Absorption}

A ratio of absorption, $\alpha(\lambda)$, is the absorbed energy ratio from incident power to the distance of the unit due to specific dissolved particles like phytoplankton, detritus, etc.

$$
\begin{aligned}
\mathrm{a}(\lambda)= & a_{w}(\lambda)+a_{c}^{0}(\lambda)\left(C / C_{C}^{0}\right)^{0.602}+a_{f}^{0} C_{f} \exp \left(f_{C} \lambda\right) \\
& +a_{h}^{0} C_{h} \exp \left(h_{C} \lambda\right)
\end{aligned}
$$

Where, $\lambda$ is the wavelength and aw is a pure water absorption coefficient (m-1). Additional relationships between $\mathrm{C}, \mathrm{Cf}$ and $\mathrm{Ch}$ are 


$$
\begin{aligned}
& \mathrm{Cf}=1.7408 * \mathrm{C} * \exp \left(0.12327\left(\mathrm{C} / \mathrm{C}_{0} \mathrm{C}\right)\right), \\
& \mathrm{Ch}=0.19334{ }^{*} \mathrm{C}^{*} \exp \left(0.12343\left(\mathrm{C} / \mathrm{C}_{0} \mathrm{C}\right)\right)
\end{aligned}
$$

$\mathrm{C}$ is the chlorophyll and its value is given in table 1. [5]

Table 1: Typical Chlorophyll Concentration

\begin{tabular}{|l|c|}
\hline \multicolumn{1}{|c|}{ Water Type } & Chlorophyll (C) \\
\hline Pure Sea & 0.005 \\
\hline Clear Ocean & 0.31 \\
\hline Coastal & 0.83 \\
\hline Turbid Harbor & 5.9 \\
\hline
\end{tabular}

\section{Scattering}

Dispersing coefficient, $b(\lambda)$, is the proportion of vitality dissipated from an episode power for each unit separation. It is the aggregate of in reverse dispersing, $b_{b}(\lambda)$, and forward dissipating coefficient, $b_{f}(\lambda)$. Dispersing is brought about by little and enormous particles. Little particles are the particles with refractive record equivalent to 1.15 , while huge particles have a refractive file of 1.03. The dissipating and backscattering coefficients are determined as follows [23]

$$
b(\lambda)=b_{w}(\lambda)+b_{s}^{0}(\lambda) C_{s}+b_{l}^{0}(\lambda) C_{l}
$$

The Back scattering coefficient for the bio optical model is a function of wavelength and chlorophyll concentration is given by [11]

$$
b_{b}(\lambda)=0.5 b_{w}(\lambda)+0.039 b_{s}^{0}(\lambda) C_{s}+6.4 \times 10^{-4} b_{l}^{0}(\lambda) C_{l}
$$

Where,

$$
\begin{aligned}
& b_{w} \text { is the pure water scattering coefficient }(\mathrm{m}-1) \text {, } \\
& b_{\mathrm{s} 0} \text { is the scattering coefficient for small particulate matter }(\mathrm{m} 2 / \mathrm{g}) \\
& b_{10} \text { is the scattering coefficient for large particulate matter }(\mathrm{m} 2 / \mathrm{g}) \text {, } \\
& C_{s} \text { is the concentration of small particles }(\mathrm{g} / \mathrm{m} 3) \text { and } \\
& C_{1} \text { is the concentration of large particles }(\mathrm{g} / \mathrm{m} 3) \text {. } \\
& C_{s}=0.01739 \text { Cc } \exp (0.11631(\mathrm{Cc} / \mathrm{C} 0 \mathrm{C})) \\
& C_{1}=0.76284 \mathrm{Cc} \exp (0.03092(\mathrm{Cc} / \mathrm{C} 0 \mathrm{C}))
\end{aligned}
$$

Small and large particulate matter and its spectral densities are given by

$$
\begin{aligned}
& b_{w}(\lambda)=0.005826(400 / \lambda)^{4.322} \\
& b_{s}^{0}(\lambda)=1.1513(400 / \lambda)^{1.7} \\
& b_{l}^{0}(\lambda)=0.3411005826(400 / \lambda)^{0.3}
\end{aligned}
$$




\section{Oceanic Turbulence}

Optical remote interchanges are extraordinarily influenced by optical disturbance, which alludes to arbitrary variances of the refraction record. On account of submerged frameworks, these vacillations are mostly brought about by varieties in temperature and saltiness of the maritime water. A significant parameter for the portrayal of maritime disturbance is the glimmer record, which communicates the change of the wave force. As appeared in Ref. [32], when a Gaussian pillar engenders through feeble disturbance and without mulling over the dispersing wonder, the shine list is communicated as the entirety of two segments

$$
\sigma_{1}^{2}(\vec{r}, L, \lambda)=\sigma_{1, l}^{2}(0, L, \lambda)+\sigma_{1, y}^{2}\left(\vec{r}_{,}, L, \lambda\right)
$$

The division among frail and solid disturbance is generally done by means of Rytov fluctuation $\sigma^{2} R$, which is the shine list when a plane wave is thought of. All the more explicitly, estimations of $\sigma^{2} R \ll 1$ compare to powerless changes, though $\sigma^{2} \mathrm{R} \gg 1$ shows a solid choppiness system. As opposed to barometrical examinations, solid maritime disturbance shows up at separations shorter than $100 \mathrm{~m}[32]$.

The blurring coefficient can be figured measurably utilizing an appropriate circulation relying upon the choppiness system. As examined in Ref. [33], the log-ordinary dispersion is utilized in circumstances of powerless changes, and its likelihood thickness work (PDF) can be composed as follows [34].

Jamali et al. Likewise proposed the blend of exponential and log-typical conveyances, making a two-projection dissemination, to more readily portray circumstances of got signals with an enormous powerful range. Such a case compares to the broad power variances of the got signal, because of the nearness of air bubbles.

\section{LINK BUDGET AND MODULATION TECHNIQUES}

\section{Link Budget}

Observational way misfortune models are sufficiently compelling to assess the got optical influence for submerged correspondences under LOS conditions. A few models showed up in the writing however the most nonexclusive is the accompanying which considers the transmitter power, telescope increase, and misfortunes [6, 19]

$$
P_{Y}=P_{t} \eta_{t} \eta_{\gamma} e^{\frac{-\mathrm{o}[\lambda] R}{\cos \theta} 2 \pi R^{2}\left(1-\cos \theta_{0}\right)}
$$

$\theta$ is the angle between the perpendicular to the Rx plane and the Tx-Rx trajectory, and $\mathrm{Ar}$ is the receiver aperture area [19].

\section{Optical Modulation Techniques}

The most straightforward and broad balance method is the aberrant tweak with direct identification (IM/DD) with on-off keying (OOK). OOK is separated into two classes, the arrival to zero (RZ) OOK and the non-come back to zero (NRZ). The articulations for the foreseen bit blunder rate (BER) are typically delivered utilizing the Poisson model for photon appearance in photon counters [6, 19].

The principle disservice of OOK balance is that it requires dynamic thresholding for the immediate discovery; something a bit much for beat position regulation (PPM). Here, the transmitter transmits a light heartbeat and makes a particular schedule opening, while the beneficiary distinguishes the beat, computes its position, and changes the sign to the 
first heartbeat [35]. PPM devours lower vitality however it has lower transmission capacity proficiency. For the L-PPM conspire, the image compares to $\mathrm{M}=\log _{2} \mathrm{~L}$ bits. Other altered PPM adjustments have been proposed, for example, differential heartbeat position balance (DPPM), double heartbeat span tweak (DPIM), and double header beat stretch balance (DH-PIM). By misusing the diverse polarization conditions of light, we can improve the presentation of SORC frameworks if there should be an occurrence of exceptional backscattering, disturbance, and surrounding light. Such a way was proposed in Ref. [36], where a paired polarization move keying (BPolSK) framework was utilized. Another regulation method with better numerical outcomes is the spellbound PPM (P-PPM) [37]. P-PPM is a mix of PPM and PolSK and figures out how to keep up the advantages of the two plans improving information rate, BER, and connection separation.

\section{RESEARCH ON SORC SYSTEMS}

Research on SORC is a very charming theme, and a few trials were completed around the world. Coming up next are some characteristic of the numerous examinations introduced in the opentechnical writing. For more data, the perusers are alluded to the total studies of Refs. [38, 39], which inspect the SORC zone in more subtleties.

An optical modem equipped for optical information pondering was structured and actualized in 2005 at Massachusetts Institute of Technology by Vasilescu et al. [54]. They utilized a LED with 532nm, 700mW brilliant force and a PIN photodiode collector with surface territory of $8 \mathrm{~mm} 2$. They utilized DPIM at a normal information pace of $320 \mathrm{~kb} / \mathrm{s}$ with 2 bits per heartbeat. A $4 \%$ parcel misfortune correspondence over $7 \mathrm{~m}$ separation and a near mistake free over $6.4 \mathrm{~m}$ through clear waters was taken note.

Two early forms of AquaOptical, that is, a lightweight gadget for high rate long range submerged point-to-point correspondence are appeared in Ref. [41]. The analysts utilized a Luxeon V LED at 470nm (480 mW) or 532nm (700mW) and a PDB-C156 photodiode in the main form. This is really a scaled down rendition equipped for blunder free correspondence in sea water at information paces of $4 \mathrm{Mbps}$ over a separation of $2.2 \mathrm{~m}(470 \mathrm{~nm})$ and $2.4 \mathrm{~m}(532 \mathrm{~nm})$.

In the subsequent one, they utilized a variety of six such LEDs and a torrential slide photodiode, and they figured out how to accomplish a BER of 0.05 at $8 \mathrm{~m}$. The improved AquaOptical II with 18 LEDs and APD at the collector was shown in 2010 . It worked at $470 \mathrm{~nm}$ with complete transmit intensity of $10 \mathrm{~W}$. Information paces of $4 \mathrm{Mb} / \mathrm{s}$ at $50 \mathrm{~m}$ in a pool were accomplished utilizing NRZ abundancy regulation with 2bits per image [42, 43]. In Ref. [44], a robot assessing its situation as per a sensor hub in an information reflecting on application-another utilization of Aquaoptical II-was depicted.

A connection spending investigation of NLOS geometries and varieties of point-to-point joins acted in 2005 and 2006 atWoods Hole Oceanographic Institution (Woods Hole MA USA), by Farr et al. [24]. Their work is just legitimate in clear waters since they don't consider spatial scattering and numerous dissipating regardless of whether they think about the weakening coefficient. Their investigation indicated that a LOS connection could accomplish $10 \mathrm{Mbps}$ at $100 \mathrm{~m}$ with $6 \mathrm{~mW}$ of transmitted force. Be that as it may, for the NLOS connect, a 50-crease increment in transmit power is important to accomplish similar information rates at comparative range because of more extensive transmit shafts. In 2008 , they accomplished transmission, a ways off of $200 \mathrm{~m}$ for an information pace of 5Mbps, and in 2010, a ways off of $108 \mathrm{~m}$ for an information pace of $10 \mathrm{Mbps}[46,47]$. In 2006, Cochenour et al. utilized BPSK with a 70-MHz radiofrequency (RF) transporter at Naval Air Fighting Center to associate mistake free correspondence at an information pace of $1 \mathrm{Mb} / \mathrm{s}$ in profound turbid water. Outflow frequency was $532 \mathrm{~nm}$ and yield power $500 \mathrm{~mW}$ [48]. The work was reached out in 2007 , to 
5Mbps with QPSK, 8-PSK, 16-QAM and 32-QAM balances and yield power 750mW [49]. Results demonstrated that even in turbid waters ( $c=3.0 / \mathrm{m}$ and $\mathrm{cz}=11$ ), no loss of regulation profundity, that is, no worldly scattering of the $70 \mathrm{MHz}$ bearer was watched, even with an enormous recipient FOV of around 100degrees. Accordingly, the main channel hindrance was because of weakening. Consequently, with enough optical force $(\sim 3 \mathrm{~W})$, a 32-QAM connect accomplished practically $5 \mathrm{Mbps}$.

Hanson and Radic [59] introduced a Monte Carlo model to anticipate the spreading of an optical heartbeat for various water types for different FOVs. They figured out how to improve the sign to clamor proportion (SNR) by expanding the FOV for low frequencies. They likewise watched worldly dispersionat high frequencies. After certain controls, they inevitably accomplished $1 \mathrm{GHz}$ transmission over a $2 \mathrm{~m}$ water pipe that reproduced seaside sea waters with $36 \mathrm{~dB}$ of lessening. Also, Jaruwatanadilok [59] attempted to foresee transient scattering by following the vector radiative exchange hypothesis to display the SORC channel which incorporates the numerous dissipating impacts and polarization. He inspected the impact of the transmission separation on channel scattering and indicated a reducing estimation of ISI for high information rates over a significant distance.

In 2008, Cochenour et al. [26], led an examination of different dissipating embellishments. They estimated the bar spread capacity tentatively in a huge test tank, by filtering a photodetector along the side to the pillar hub after the shaft has engendered some separation. Results demonstrated that in clear waters, the power tumbles off quickly with sidelong good ways from the shaft. Be that as it may, their estimation disregarded any effect of transient scattering. In another examination on spatial scattering, they explored the effect of ingestion on forward dissipated light [51].

Later the creators additionally propelled a set up which estimated the transmission of light adjusted at frequencies up to $1 \mathrm{GHz}$ in reenacted sea water up to 55 weakening lengths [52-54].

At long last, the creators analyzed a retro-reflecting connection and figured out how to decrease the commitment of backscattered light utilizing polarization separation strategies [55].

A test led in North Carolina State University where a $405 \mathrm{~nm}$ laser with OOK was tried in a water tank of 3.66m long. From the start, a dependable transmission at 500kbps was accomplished [56]. A few years after the fact, a duplex correspondence at $1 \mathrm{Mbps}$ was achieved [57]. In addition,

Simpson et al. Made two isolated optical light emissions with two transmitters and two beneficiaries joined with a pillar splitter [58]. In a later work, they introduced two set ups, one in $3 \mathrm{~m}$ through turbid water and the other over a length of $7.7 \mathrm{~m}$ through less turbid water and accomplished 5Mbps throughput [59].

Dalgleish et al. [64] built up a Monte Carlo recreation technique to assess motivation reactions for a specific framework equipment plan over an enormous scope of natural conditions in turbid sea situations. Besides, an incredible asset to assess the presentation of a submerged framework for self-ruling submerged vehicle interchanges was introduced in Ref. [61]. Trial tests were likewise completed. Equipment modules and circuits plan for submerged optical point to point joins are additionally examined in Ref. [62].

NLOS plans where optical bars are reflected in the air water interface were talked about in Ref. [6]. These geometries have the advantage to maintain a strategic distance from intermittent obstacles yet they don't have a similar exhibition as LOS joins. Gabriel et al. [27] accepted a Monte Carlo model of a submerged channel and reenacted the directions of the produced photons. They thought about a few parameters, for instance, the transmitter pillar width and bar 
difference, shaft frequency, connect separation, FOV, water types and turbidity, and opening size. They arrived at the resolution that the channel postpone spread is about zero when the dispersing albedo is moderate in little separations.

In the most recent years, the examination is centered around spatial assorted variety, as an approach to improve by and large the exhibition defiled by choppiness. This is normally cultivated by including more sources or indicators. Additionally, opening averaging strategies were proposed. In Ref. [63], Yi et al. Executed a gap averaging procedure on the glitter file for either planeor round waves, so as to destroy the negative impacts of blurring coming about because of maritime disturbance. A different info single-yield (MISO) interface was portrayed in Ref. [64], where the source is supplanted by a uniform roundabout exhibit of sources, and the photons are gathered by a single indicator. The numerical outcomes demonstrated a debasement in BER, under choppiness conditions. A solitary information various yield (SIMO), comprising of a LED as a source and a concentric roundabout cluster of focal points as a locator, was talked about in Ref. [65]. The framework figures out how to broaden the successful correspondence go by about $20 \mathrm{~m}$, even on account of solid disturbance.

A progressively broad set up of the various information numerous yield (MIMO) frameworks was introduced in Ref. [66]. At long last, in Ref. [67], a spatial assorted variety method in mix with optical intensification to additionally improve execution was proposed.

\section{CONCLUSIONS}

The need to give outpost remote interchanges to submerged applications will be expanding in the inevitable years. SORC comprises a substitute and viable transmission method which can achieve this extension, rather than the customary acoustic one. Because of this reality, a few examinations on SORC frameworks were contrived worldwide in the ongoing years. The current investigation gave a brief survey of the key advances in channel displaying and exploratory works detailed so far in the specialized writing.

\section{REFERENCES}

1. Balaji K, Dr. S. Sakthivel Murugan, (2019), "Implementing IoT in Underwater communication using Li-Fi", International Journal of Recent Technology and Engineering (IJRTE) ISSN: 2277-3878, Volume-8 Issue-2S4, July 2019. DOI: 10.35940/ijrte.B1190.0782S419

2. B.Dheepa, R.Nithya, N.Nishavithri, K.Vinoth, K.Balaji (2020), "Directional lifting wavelet transform based SAR Image Compression," International Journal of Advanced Trends in Computer Science and Engineering, Volume-9, No.3, May-June 2020. https://doi.org/10.30534/ijatcse/2020/183932020

3. Urick Robert J., (1983), "Principles of underwater sound", Book, Peninsula Publishing, Science, Los Altos CA

4. Li B., Stojanovic M., Freitag L., and Willett P., (2008), "Multicarrier communication over underwater acoustic channels with nonuniform Doppler shifts". IEEE Journal of Oceanic Engineering, Vol. 2, No. 33, pp. 198-209

5. Kilfoyle D. B., and Baggeroer A. B., (2000), "The state of the art in underwater acoustic telemetry", IEEE Journal of Oceanic Engineering, Vol. 25, No. 1, pp. 4-27

6. Stojanovic M.,(2003) "Acoustic (underwater) communications", in J.G. Proakis(Ed.), Encyclopedia of Telecommunications, Wiley, New York.

7. Pelekanakis C., Stojanovic M., Freitag L., (2003), "High rate acoustic link for underwater video transmission", OCEANS 2003, 22-26 September 2003, Vol. 2, pp. 1091-1097 
8. Pei L.V., Junhua H., Renkui Z., Haiying L., (2008), "The application of underwater optics and its development”, Information Optics and Photonics Technologies II, Vol. 6837, pp. 7-8

9. Lanbo L., Shengli Zhou, and Jun-Hong Cui, (2008) "Prospects and problems of wireless communication for underwater sensor networks", Wireless Communications and Mobile Computing, Vol. 8, No. 8, pp. 977-994

10. Vavoulas A., Sandalidis H.G, and Varoutas D., (2012) “Weather effects on FSO network connectivity”, Journal of Optical Communications Networking, Vol. 4, No. 10, pp. 734- 740.

11. Young E.Y.S, Bullock A.M., (2003), "Underwater airborne laser communication system characterization of the cannel" Free-Space Laser Communication Technologies XV, G. Stephen Mecherle, Editor, Proceedings of SPIE, Vol. 4975

12. Haltrin V., (1999) “Chlorophyll-based model of seawater optical properties”, Applied Optics, Vol. 38, No. 33, pp. 6826-6832

13. Cochenour B., (2013) "Experimental measurements of temporal dispersion for underwater laser communications and imaging”, PhD Thesis, North Carolina State University USA

14. Gabriel C., Khalighi M.A., Bourennane S., Pierre Leon, Vincent Rigaud, (2013) "Montecarlo- based channel characterization for underwater optical communication systems" Journal of Optical Communications and Networking January 2013, Vol. 5, Issue 1, pp. 1-12 The Optical Society

15. Arnon S., (2003), “Optical wireless communications”, Book Chapter, Encyclopedia of Optical Engineering (EOE), Edited by R. G. Driggers, 3 Marcel Dekker, pp. 1866-1886

16. Cochenour B., Mullen L., Laux A., (2008), “Characterization of the beam-spread function for underwater wireless optical communications Links", IEEE Journal of Oceanic Engineering, Vol. 33, No.4, pp. 513-521

17. Andrews L.C. and Phillips R.L., (2005) Laser Beam Propagation through Random Media, Date Published: 16 September 2005, Pages: 808, ISBN: 9780819459480, Volume: PM152Published by SPIE The International Society of Optical Engineering, Bellingham.

18. Meihong S., Xinsheng Y. and Fengli Z., (2009), "The evaluation of modulation techniques for underwater wireless optical communications", International Conference on Communication Software and Networks, Chengdu Sichuan China, 27-28 February 2009, pp. 138-142

19. Dong Y., Zhang T., and Zhang X., (2013) "Polarized pulse position modulation for wireless optical communications", 2013 47th Annual Conference on information Sciences and Systems (CISS), Baltimore Maryland, 20-22 March 2013

20. Zeng Z., Zhang H., Dong Y, and Cheng J., (2017) “A Survey of underwater wireless optical communication,” IEEE Communication. Survey Tutorials, to appear

21. Doniec M., Vasilescu I., Mandar C., Detweiler C., Hoffmann-Kuhnt M., Rus D., “AquaOptical: A Lightweight Device for High-rate Long-range Underwater Point-to-Point Communication” Marine Technology Society Journal, Vol. 44, No. 4, pp. $55-65$

22. Doniec M. and Rus D., (2010), "Bidirectional optical communication with AquaOpticallI," in Communication Systems (ICCS), 2010 IEEE International Conference on, Nov. 2010, pp. 390-394

23. Farr N., Chave A., Freitag L., Preisig L., White S., Yoerger D., and Sonnichsen F., (2006), “Optical modem technology for seafloor observatories”, OCEANS 2006, Singapore, 16- 19 May 2006

24. Farr N., Ware J., Pontbriand C., Hammar T., Tivey M.,(2010) “Optical communication system expands cork seafloor observatory's bandwidth", OCEANS 2010, Sydney Australia, 24-27 May 2010 
25. Cochenour B., Mullen L., and Laux A., (2007) "Phase coherent digital communications for wireless optical links in turbid underwater environments, " OCEANS 2007, Aberdeen Scotland UK, 18-21 June 2007

26. Cochenour B., Mullen L., and Muth J., (2010) "Effect of scattering albedo on attenuation and polarization of light underwater", Optics Letters, Vol. 35, No. 12, pp. 2088-2090

27. Mullen L.J., Laux A.E., Cochenour B.M., (2009) "Propagation of modulated light in water: Implications for imaging and communications systems", Applied Optics, Vol. 48, No. 14, pp. 2607-2612

28. Mullen L., Cochenour B., RabinovichW., Mahon R., Muth J., (2009) “Backscatter suppression for underwater modulating retroreflector links using polarization discrimination”, Applied Optics, Vol. 48, No. 2, pp. 328-337

29. Cox W., Simpson J., and Muth J., (2011), "Underwater optical communication using software defined radio over led and laser based links, "Military Communications Conference, 2011 MILCOM 2011, Baltimore MD USA, pp. 2057-2062

30. Simpson J, CoxW., Krier J, Cochenour B., Hughes B., and Muth J., (2010), "5 Mbps optical wireless communication with error correction coding for underwater sensor nodes" Sydney Australia, 24-27 May 2010

31. Anguita D., Brizzolara D., Parodi G., and Hu Q., (2011), “Optical wireless underwatercommunication for AUV: Preliminary simulation and experimental results”, OCEANS2011, Waikoloa HI USA, 19-22 September 2011

32. Yi X., Li Z., and Liu Z., (2015) "Underwater optical communication performance for laser beam propagation through weak oceanic turbulence”, Applied Optics, Vol. 54, No. 6, pp. 1273, 2015

33. Liu W., Xu Z., and Yang L., (2015) "SIMO detection schemes for underwater optical wireless communication under turbulence", Photonics Research, Vol. 3, No. 3, pp. 48-53

34. Boucouvalas A., Peppas K., Yiannopoulos K., and Ghassemlooy Z., (2016) “Underwater optical wireless communications with optical amplification and spatial diversity, ” IEEE Photonics Technology Letters, Vol. 28, No. 22, pp. 2613-2616

35. Vall L.D., (2011), Towards Underwater Video Transmission, MSc Thesis, Massachusetts Institute of Technology. https://seagrant.mit.edu/publications/

36. Stojanovic M., (2008), “Underwater acoustic communications: Design considerations on the physical layer”, IEEE/IFIP Fifth Annual Conference onWireless On demand Network Systems and Services (WONS 2008), Garmisch-Partenkirchen Germany, January 2008, pp. 2-3

37. Arnon S. and Kedar D., (2009), "Non-line-of-sight underwater optical wireless communication network", Journal of the Optical Society of America A, Vol. 26, No. 3, pp. 530-539

38. Akyildiz I.F., Pompili D., and Melodia T., (2005), "Underwater acoustic sensor networks: Research challenges", Ad Hoc Networks, Vol. 3, No. 3, pp. 257-279

39. Glegg S.A.L., Pirie R., LaVigne A.,(2000) “A study of ambient noise in shallow water”, Florida Atlantic University Technical Report

40. Stojanovic M., (1996), “Recent advances in high-speed underwater acoustic communications”, IEEE Journal of Oceanic Engineering, Vol. 21, No. 2, pp. 125-136

41. Balanis C. A., (1989), Advanced Engineering Electromagnetics, John Wiley \& Sons, New York, NY.

42. Gkoura L.K., Nistazakis H.E, Vavoulas A., Tsigopoulos A.D., and Tombras G.S., (2014) "Underwater optical wireless communications possibilities disadvantages and possible solutions" 6th International Conference from Scientific Computing to Computational Engineering IC-SCCE, 9-12 July Athens 
43. Andrews L.C., Phillips R.L., and Hopen C.Y.,(2001), Laser Beam Scintillation with Applications, SPIE Optical Engineering Press The International Society of Optical Engineering Bellingham Washington USA

44. Nistazakis H.E., Karagianni E.A., Tsigopoulos A.D., Fafalios M.E., and Tombras G.S., (2009) “Average capacity of optical wireless communication systems over atmospheric turbulence channels", Journal of Lightwave Technology, Vol. 27, No. 8, pp. 974-979

45. Sathyendrenath S.,(1984) "Inherent optical properties of natural seawater”, Defence Science Journal, Vol. 34, No. 1, pp. 34, $1-18$

46. Cochenour B., and Mullen L., (2012) “Free-space optical communications underwater", in Advanced Optical Wireless Communication Systems, (ed). S. Arnon, J.R. Barry, G.K. Karagiannidis, R. Schober, and M. Uysal, Advanced Optical Wireless Communication Systems Pages 273-302 Cambridge, UK: Cambridge Univ. Press

47. Gupta S.K.,(1984) "Spectral transmission studies of ocean water under different sea conditions", Defence Science Journal, Vol. 34, No. 1, pp. 19-28

48. Mobley C.D., (1994), "Light and Water: Radiative Transfer in Natural Waters", Book, Academic Press Inc. A division of Harcourt Brace and Company 525B Street Suite 1900, San Diego, California 92101-4495 United Kingdom Edition published by: Academic Press Limited, 24-28 Oval Road, London NW1 7DX

49. Hanson R., and Radic S,(2008) "High bandwidth underwater optical communication", Applied Optics, Vol. 47, No. 2, pp. $277-283$

50. Korotkova O., Farwell N., and Shchepakina E., (2012) "Light scintillation in oceanic turbulence", Waves in Random and Complex Media, Vol. 22, No. 2, pp. 260-266

51. Jamali M.V., Khorramshahi P., Tashakori A., Chizari A., Shahsavari S., Abdollah-Ramezani S., Fazelian M., Bahrani S., and Salehi J.A., (2016) "Statistical distribution of intensity fluctuations for underwater wireless optical channels in the presence of air bubbles", Iran Workshop on Communication and Information Theory IWCIT 2016, 3-4 May 2016

52. Cox W.C., Hughes B.L, and Muth J.F., (2009) “A polarization shift-keying system for underwater optical communications”, OCEAN 2009, Biloxi MS USA, 26-29 October 2009

53. Kaushal H. and Kaddoum G., (2016), “Underwater optical wireless communication”, IEEE Access, Vol. 4, pp. 1518-1547

54. Vasilescu I., Kotay K., Rus D., Dunbabin M., Corke P., (2005), "Data collection, storage, and retrieval with an underwater sensor network", ACM Proc. 3rd International Conference on Embedded Networked Sensor Systems (SenSys), San Diego California USA, 2-4 November 2005, pp. 154-165

55. Doniec M., Detweiler C., Vasilescu I, and Rus D., (2010), "Using optical communication for remote underwater robot operation”, 2010 IEEE/RSJ International Conference on Intelligent Robots and Systems (IROS), Taipei Taiwan, 18-22 October 2010, pp. 4017-4022

56. Doniec M.W., Topor I., Chitre M., and Rus D.,(2012), “Autonomous, localization-free underwater data muling using acoustic and optical communication”, The 13th International Symposium on Experimental Robotics ISER 2012, Québec City, Canada, June 18-21 2012

57. Pontbriand C., Farr N., Ware J., Preisig J., Popenoe H., (2008) "Diffuse high-bandwidth optical communications", OCEANS 2008, Quebec City QC, Canada, 15-18 September 2008

58. Cochenour B., Mullen L., Laux A., and Curran T. (2006), "Effects of multiple scattering on the implementation of an underwater wireless optical communications link, "Singapore, 16-19 May 2006 
59. Jaruwatanadilok S., (2008), "Underwater wireless optical communication channel modeling and performance evaluation using vector radiative transfer theory”, IEEE Journal on Selected Areas in Communications, Vol. 26, No. 9, pp. 1620-1627

60. Mullen L., Alley D., Cochenour B., (2011) 'Investigation of the effect of scattering agent and scattering albedo on modulated light propagation in water' Applied Optics Vol. 50, Issue 10, pp. 1396-1404. https://doi.org/10.1364/AO.50.001396

61. Mullen L., Cochenour B., Laux A., and Alley D., "Optical modulation techniques for underwater detection, ranging, and imaging”, In Proc. of SPIE Ocean Sensing and Monitoring III, pp. 1-9. SPIE, 2011

62. Cox W., (2008), “A 1Mbps underwater communication system using a 405nm laser diode and photomultiplier tube,” MSc Thesis, North Carolina State University USA

63. Simpson J., Hughes B., and Muth J., (2009), "A spatial diversity system to measure optical fading in an underwater communications channel," OCEANS 2009, Biloxi MS USA, 26-29 October 2009

64. Dalgleish F.R., Caimi F.M., Vuorenkoski A. K., Britton W.B., Ramos B, (2010), "Efficient laser pulse dispersion codes for turbid undersea imaging and communications applications", Proc. SPIE, Vol. 7678

65. Anguita D., Brizzolara D., and Parodi G., (2010), "Optical wireless communication for underwater wireless sensor networks: Hardware modules and circuits design and implementation”, OCEANS 2010, Sydney Australia, 24-27 May 2010

66. Dong Y. and Liu J., (2016) "On BER performance of underwater wireless optical MISO links under weak turbulence”, OCEANS 2016, Monterey CA USA, 19-23 September 2016

67. Jamali M.V. and Salehi J.A., (2015) "On the BER of multiple-input multiple-output underwater wireless optical communication systems, " 4th International Workshop on Optical Wireless Communications IWOW 2015, Istanbul Turkey, 0708 September 2015, pp. 26-30

68. Mahdi, A., F. Al-Khudair, and A. Subber. "Identification of mixed symmetry state in 180-186 W isotopes in framework of IBM2." International Journal of Physics and Research (IJPR) 4.5 (2014): 1-12.

69. Kaur, Navdeep, and Harmeet Singh. "A Survey on Recent Work in Hybrid FSO/RF Communication Link." International Journal of Electronics, Communication \& Instrumentation Engineering Research and Development (IJECIERD) 9.1 (2019) : 25-32.

70. Patel, P R, and D J Shah. "Smart Diagnostic System for Classification of Diabeticretinopathy using Image Processing Techniques." IASET: International Journal of Electronics and Communication Engineering (IJECE) 5.5 (2016) : 49-58.

71. Raj, N. Gokul. "A Fair Channel Scheduling in Multi Channel Multi Interface Wireless Mesh Networks." International Journal of Electronics and Communication Engineering (IJECE) 2.3 (2013) : 33-40/

\section{AUTHOR DETAILS}

- Mr. Balaji $\mathbf{K}$ is working as an Assistant professor in the department of Electronics and Communication Engineering at Mailam Engineering College, Mailam, Tamilnadu. He is doing doctorate in the area of underwater optical communication.

- Ms.Nithya $\mathbf{R}$ is working as an Assistant professor in the department of Electronics and Communication Engineering at Mailam Engineering College, Mailam, Tamilnadu.She is doing research in the area of underwater optical communication. 
- Dr.S.Ganesan is working as an Professor and HoD of ECE Department at Mailam Engineering college, Mailam. He has teaching as well as industry experience of more than three decades. His area of research is wireless networks, wireless communication, etc.,

- Mrs. N. Nishavithri is working as an Assistant professor in the department of Electronics and Communication Engineering at Mailam Engineering College, Mailam , Tamilnadu. She is doing research in the area of underwater optical communication.

- Dr.S.Sakthivel Murugan is working as an Associate professor in the Department of ECE at SSN college of Engineering, Chennai. His major research area is Underwater Image processing, Underwater signal Processing, Underwater optical communication. 